\title{
Neutrino Telescope Array (NTA): Prospect towards Survey of Astronomical $v_{\tau}$ Sources
}

\section{George Wei-Shu Hou*}

Department of Physics, National Taiwan University, Taipei 10617, Taiwan

E-mail: wshou@phys.ntu.edu.tw

\section{Makoto Sasaki}

Institute for Cosmic Ray Research, University of Tokyo, Kashiwa, Chiba 277-8582, Japan

E-mail: sasakimeicrr.u-tokyo.ac.jp

\begin{abstract}
The recent observation of astrophysical PeV neutrino events by IceCube opens a new chapter in neutrino astronomy, calling for improved point-back abilities and increased sensitivity to fill the gap between IceCube and Auger and probe for $\mathrm{PeV}-\mathrm{EeV}$ neutrino point sources. By separating $v_{\tau} \rightarrow \tau$ conversion from $\tau$-shower generation, the Earth-skimming $v_{\tau}$ method allows for huge target mass and detection volume simultaneously. The Ashra-1 detector, in operation up Mauna Loa on Hawaii Big Island, has demonstrated the feasibility in a $1^{\text {st }}$ search for $v_{\tau}$ from a GRB. With design based on this experience, a Neutrino Telescope Array (NTA) observatory is being planned: it will have three site stations watching the air mass surrounded by Mauna Loa, Mauna Kea, and Hualalai, plus a central site station watching the lower night sky. Sensitivities equivalent to $>100 \mathrm{~km}^{3}$ water and pointing accuracy of $<0.2^{\circ}$ can be achieved with Cherenkov-fluorescence stereoscopic observation for $\mathrm{PeV}-\mathrm{EeV}$ neutrinos that is almost background-free. With the scientific goal of clear discovery and identification of astronomical $v_{\tau}$ sources, a new International Collaboration is being formed to probe for cosmic proton accelerators.
\end{abstract}

The 34th International Cosmic Ray Conference,

30 July- 6 August, 2015

The Hague, The Netherlands

\footnotetext{
* Speaker.
} 


\section{Introduction: towards $v$-Astronomy}

Recently, IceCube announced [1] the observation of three PeV scale astrophysical neutrino events: at $1.04 \pm 0.16 \mathrm{PeV}$ ("Bert"), $1.14 \pm 0.17 \mathrm{PeV}$ ("Ernie"), and $\sim 2 \mathrm{PeV}$ ("Big Bird"). They beg the question: What if one had better sensitivity and accurate pointing? Based on the concepts and development of the NuTel and Ashra-1 experiments [2], we propose a new experiment, the Neutrino Telescope Array (NTA), with scientific goal of Clear Discovery and Identification of Nonthermal Hadronic Processes in the Universe, be it Galactic, Extragalatic, or Cosmogenic.

Neutrino astronomy started with the quest by Ray Davis for solar neutrinos, where he discovered the solar neutrino deficit and stumbled upon large neutrino mixing, $\theta_{12}$. The KamiokaNDE detector, based on water Cherenkov detection of $v_{e}$-scattered electrons and for purpose of nuclear decay detection, lead to imaging the Sun from underground! It confirmed the Davis effect, but its directional and timing abilities serendipitously detected neutrinos from SN1987A from the Large Magellanic Cloud, and neutrino astronomy turned extra-galactic. Subsequently, Super-K discovered atmospheric neutrino effect [3], unveiling near-maximal $\theta_{23}$.

In watching stars, David the Shepherd once uttered in wonderment: "Their lines go out through all the Earth!" (Psalm 19) David certainly did not know about Cosmic Rays (CR), but especially neutrinos, chargeless and weakly interacting, fulfill his poetic prophecy, and point back to far away sources. Counting solar and SN neutrinos as "done", and atmospheric neutrinos as "nuisance", our next objective is the detection of cosmic neutrinos from very far away sources. Because of the rapid fall-off of the CR flux with energy, these would have to be extremely energetic. Thus, to catch the low flux, current and developing neutrino telescopes are all of " $\mathrm{km}^{3}$ " size or beyond. The leading detector is IceCube [4], with a full $\mathrm{km}^{3}$ of pristine ice instrumented with more than five thousand 10 inch PMTs. The full detector was completed at end of 2010, and the aforementioned PeV neutrino announcement has turned our eyes towards very high energy (VHE) neutrino astrophysics.

The VHE $v$-astrophysics can become $v$-astronomy if one has improved point-back ability and accumulate more events, which is the purpose of the NTA. VHE neutrinos are produced when protons are accelerated and collide with radiation fields or the ambient matter, i.e.

$$
p+\gamma \rightarrow \Delta^{+} \rightarrow \pi^{0}+p, \pi^{+}+n ; \quad p+\text { nucleus } \rightarrow \pi^{ \pm, 0}+X .
$$

Secondary neutrinos from $\pi^{ \pm}$decay would indicate proton acceleration and point to the accelerators. Secondary $\gamma$ rays from $\pi^{0}$ would imply more nearby sources. The search for both $v$ s and $\gamma_{s}$ would explore the accelerators in the Universe in a multi-messenger approach. Possible extragalactic sources are active galactic nuclei (AGN), $\gamma$-ray bursts (GRB), and starburst galaxies (SBG).

The current leading detector for ultra high energy cosmic rays (UHECR), at and above $10^{18} \mathrm{eV}$ energies, is Auger [5]. Covering $\sim 3000 \mathrm{~km}^{2}$, the detector was completed in 2008. One of the main targets is the so-called GZK [6] neutrinos, which arise through the $\Delta$-conversion of UHE $\left(10^{19} \mathrm{eV}\right.$ or above) cosmic protons by interaction with the cosmic microwave background (CMB) radiation, a process similar to Eq. (1.1). It is necessarily diffuse. But contrasting this UHECR approach using water tanks and fluorescence detectors, with the IceCube approach of water Cherenkov detection of cosmic neutrinos, we note that the gap or window between $\mathrm{PeV}$ to $\mathrm{EeV}\left(10^{15} \mathrm{eV}\right.$ to $\left.10^{18} \mathrm{eV}\right)$ still lacks a dedicated experiment, which is quite amazing. The discovery of PeV neutrinos by IceCube suggests a supra-PeV neutrino spectrum, making it imperative to explore this region in earnest. 


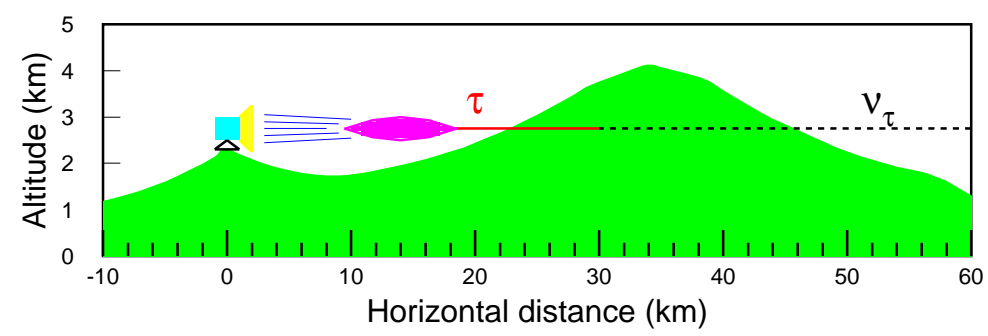

Figure 1: Illustration of $v_{\tau} \rightarrow \tau$ conversion inside a mountain and the detection technique [7]. The projection is a slice through Hualalai and Mauna Loa of Hawaii Big Island.

Employing the so-called Earth-skimming $v_{\tau}$ method that targets the PeV to EeV region, the greater sensitivity and pointing accuracy of NTA could bring us into the $v$-astronomy era.

\section{Earth-skimming $v_{\tau}$ Method}

The Earth-skimming (ES) $v_{\tau}$ detection method combines quite a few amazing facts:

- Cosmic hadron acceleration implies $v_{\tau}$ flux: Pions produced via Eq. (1.1) should have $v_{e}$ : $v_{\mu}: v_{\tau}$ ratio of $1: 2: 0$ at production, but turns into $1: 1: 1$ at cosmic distances by near maximal $v_{\mu}-v_{\tau}$ mixing as implied by atmospheric neutrino effect [3].

- Unique to $v_{\tau}$ : With cross section growing with energy, VHE $v_{\tau}$ s can convert to $\tau$ (a MIPs) in the Earth or a mountain, and upon emerging it would decay and shower in air. For $v_{e}$, the electron shower is absorbed in mountain/Earth, while for $v_{\mu}$, the muon does not shower.

- A UV telescope placed on a mountain a few $10 \mathrm{~km}$ downstream can catch the Cherenkov pulse from the $\tau$ shower (hence the name "NuTel" [7]).

- Further advantages of the method are: mountain/Earth as shield of cosmic ray secondaries, precise arrival direction determination, and negligible atmospheric neutrino background.

The magic works for two large mountains separated by a wide valley (see Fig. 1). These points were raised around 2000 [8] (see also Refs. [7] and [9]), but brought to our attention by Vannucci [10] in 2001, and through which we realized [2] the excellent site of Hawaii Big Island: Mauna Loa and Mauna Kea each at $4000 \mathrm{~m}$. As excellent sites for astronomy, they are perfect for placing the UV telescope up $2000 \mathrm{~m}$ or higher. Mount Hualalai at $2521 \mathrm{~m}$ offers a third site [7].

The NuTel project [7] also illustrates the three stages for event simulation:

1. Mountain: $v_{\tau} \rightarrow \tau$ conversion cross section, inelasticity, energy loss;

2. Shower: $\tau$ decay modes, detailed vs fast simulation;

3. Detector Performance: light propagation and quantum efficiency, trigger, reconstruction. 


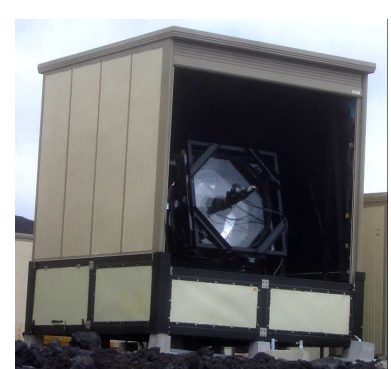

Figure 2: An Ashra-1 light collector.

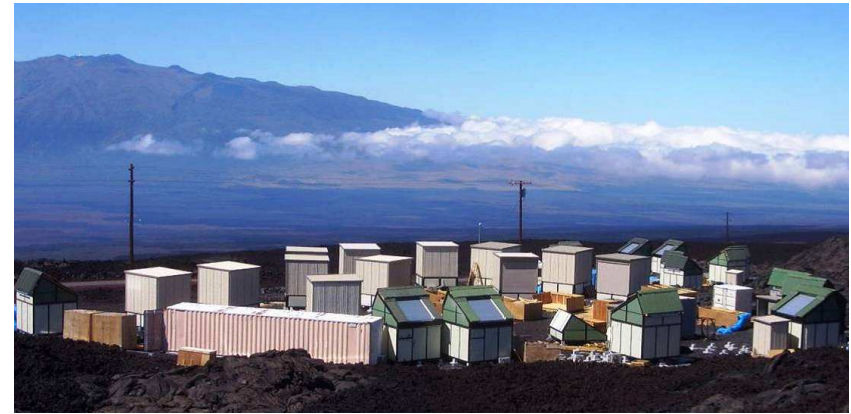

Figure 3: The Ashra-1 main and sub stations at Mauna Loa site.

With two telescopes providing stereo view, coincidence trigger, the angular error was $<1^{\circ}$, the energy resolution was decent, and reconstruction efficiency was good when triggered.

Though electronics was quickly built, optics was a challenge, and discussions went on to adopt the optical system (Fig. 2) from Ashra, where the Earth-skimming $v_{\tau}$ method was also proposed [11] with a fluorescence telescope array, which would be the basic technique Neutrino Telescope Array, as we will discuss. The NuTel proposal, however, was rejected in 2004, mainly due to an estimated low event rate of 0.5 event/year. The optics, however, was developed further, and two $1.8 \mathrm{~m}$ Schmidt mirror systems were eventually built and tested (in 2009) up $2100 \mathrm{~m}$ in Taiwan.

\section{Ashra-1 and $1^{\text {st }}$ Search for GRB $v_{\tau}$}

The All-sky Survey High Resolution Air-shower detector [12] Phase I, or Ashra-1 (see Fig. 3), was developed slightly earlier but in a similar time frame as NuTel, with the ambitious aim of "multi-messenger astronomy" [13, 14], i.e. optimized for the detection of VHE particles. In the early 1990s (Fly's Eye), optical air shower detectors had $4^{\circ}$ per PMT with all-sky coverage, and $1^{\circ}$ per PMT with $28^{\circ}$ coverage per telescope by the late 1990s (HiRes). Ashra-1 has 1.2 arcmin per pixel with potential for all-sky coverage. The key technical feature rests on the use of electrostatic lenses to generate convergent beams, rather than just optical lens systems. This enables the realization of high resolution over a wide field of view (FOV). The electron optics requires:

- Wide angle high precision optics [15]: a Schmidt type mirror with modified Baker-Nunn optics allows a compromise between very wide FOV and 1 arc min resolution on the focal sphere of the light collector (Fig. 2), with pupil diameter of $1 \mathrm{~m}$;

- Photoelectric Lens Imaging (PLI) tube [16]: the world's largest imaging-intensifier (I.I.) tube uses electrostatic lens to converge beams from 20 inch photocathode onto 1 inch output focal surface, enabling a very low cost and high performance image sensor; and

- Photoelectric Image Pipeline [17]: splits the PLI image and transport to multiple trigger devices and $\mathrm{CCD}+\mathrm{CMOS}$ pixel sensors with high gain, enabling very fine images with parallel self-trigger systems for optical (starlight), fluorescence $(\mu \mathrm{s})$ and Cherenkov (ns) emissions. 

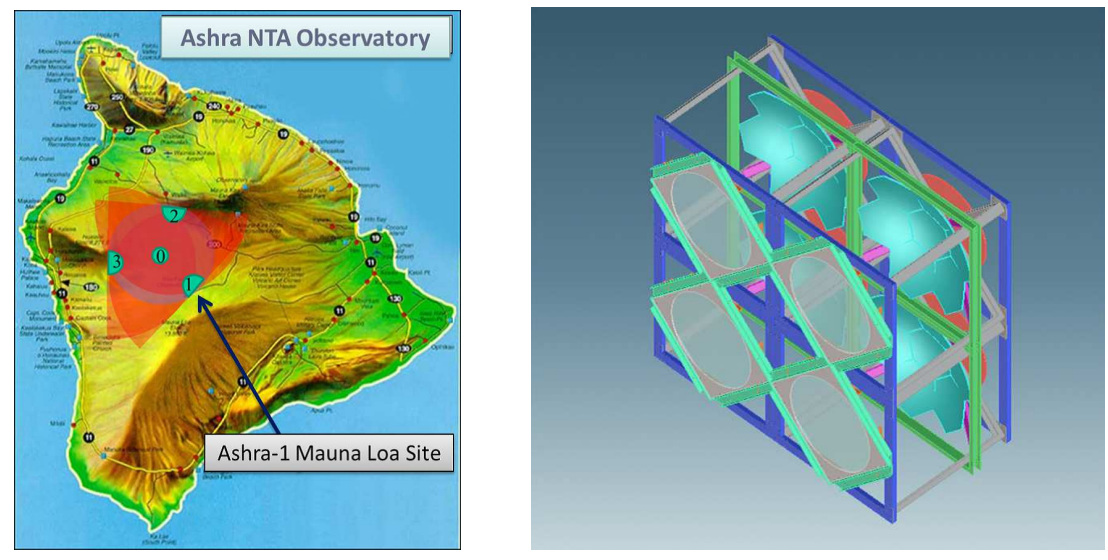

Figure 4: (left) NTA observatory layout on Hawaii Big Island, where overlay of semi-circles illustrate view from Site1-3; (right) Detector Unit (DU) of 4 same Light Collectors (LCs) scaled up from Ashra-1 design.

The Ashra-1 demonstration phase has been running since 2008 at the Mauna Loa Observation Site (ML-OS), 3300 m a.s.l. on Hawaii Big Island. With alert for GRB081203A given by SWIFT satellite, Ashra-1 succeeded in the first search for PeV $v_{\tau}$ s originating from a GRB [18] with the ES $v_{\tau}$ technique using one LC. Moreover, Ashra-1 has achieved the best-yet instantaneous sensitivity in the $100 \mathrm{PeV}$ energy region subsequent to a January 2012 trigger upgrade [19].

Ashra-1 continues with technical improvement and demonstration, such as fluorescence detection and multi-messenger exploration of PeV-EeV Universe. With the new impetus of PeV astro- $v$ observation by IceCube, let us now unfold the NTA project, the synergy of Ashra-1 and NuTel.

\section{Neutrino Telescope Array (NTA): Detector and Performance}

Based on Ashra-1 performance, we aim at forming a new collaboration, Neutrino Telescope Array (NTA), an air shower imaging neutrino detector for our stated scientific goal. With better than $0.2^{\circ}$ pointing accuracy, NTA would be able to discern the origins of IceCube PeV neutrino events. A Letter of Intent (LOI) is at hand [20], which is still being improved.

\section{$\underline{\text { Site Plan and Size }}$}

As shown in Fig. 4 (left), the planned NTA observatory consists of four sites: Site0 and Site13 , where the $x-y-z$ coordinates and corresponding FOV coverage are given in Table 1. Site1-3 are the vertices of an equilateral triangle with $25 \mathrm{~km}$ sides, and observe the total air mass surrounded by Mauna Kea, Mauna Loa, and Hualalai; the central Site0 can potentially have full-sky coverage.

VHE $v_{\tau} \mathrm{s}$ can convert in Earth/mountain, reappear as $\tau \mathrm{s}$ and produce air showers upon decay in the atmosphere, and the Cherenkov photons are detected [7, 8, 9] (see Fig. 1). By separating $v_{\tau} \rightarrow \tau$ conversion from subsequent air shower generation, detection is possible while preserving the huge target mass required for the initial interaction. Currently, Ashra-1 operates on Site1, or ML-OS, with view of Mauna Kea, which is a mountain equivalent to $10^{4} \mathrm{~km}^{3}$ of ice for converting $v_{\tau}$ to $\tau$, but it also serves as a shield to CR background. The distance between Mauna Kea and Loa allows a $30 \mathrm{~km}$ range for shower development. Ashra-1 has demonstrated [18] the ES- $v_{\tau}$ technique 


\begin{tabular}{cccccc}
\hline Site ID & Location & X $[\mathrm{km}]$ & Y $[\mathrm{km}]$ & $\mathrm{Z}[\mathrm{km}]$ & FOV [sr] \\
\hline Site0 & Center & 0.000 & 0.00 & 2.03 & $\pi$ \\
Site1 & Mauna Loa & 9.91 & -10.47 & 3.29 & $\pi / 2$ \\
Site2 & Mauna Kea & 4.12 & 13.82 & 1.70 & $\pi / 2$ \\
Site3 & Hualalai & -14.02 & -3.35 & 1.54 & $\pi / 2$ \\
\hline
\end{tabular}

Table 1: The $x-y-z$ coordinates and FOV coverage of NTA sites, where z-coordinates are from topography data and y-axis points north. Site1-Site3 form an equilateral triangle, with Site0 at center, or origin. Site1 is at ML-OS (on Mauna Loa), with Site 2 at $25 \mathrm{~km}$ distance in the direction of Kilohana Girl Scout Camp.

with this configuration, but NTA would augment it with fluorescence [11] ability of Site0, plus two other mountain sites. The huge target mass $\left(>100 \mathrm{~km}^{3}\right.$ ), huge atmospheric mass (shower volume, with area $>1000 \mathrm{~km}^{2}$ ) and mountain as background shield imply a rather large footprint for NTA, and conceptually quite different from IceCube and Auger.

Each site has a group of DUs each composed of 4 LCs (Fig. 4 (right)). The (segmented) mirrors of NTA LC scale up from Ashra- 1 by 1.5, but with same trigger and readout. It uses Schmidt optics with pupil of $1.5 \mathrm{~m}$, and FOV of $32^{\circ}$ means $50 \mathrm{~cm}$ at focal sphere. $4 \mathrm{LCs}$ with same FOV and superimposed image give a DU with effective pupil size of $3 \mathrm{~m} .12$ DUs are needed per $\pi$ solid angle coverage, so from Table 1, at least 30 DUs are needed. The construction, deployment and operation of these DUs at four distant sites call for an International Collaboration.

Pointing Accuracy

Rooted in high pixelization and the ES- $v_{\tau}$ method, pointing accuracy is a main strength of NTA. Performance studies are based on Ashra-1 experience, and more detail can be found in the LOI [20]. Detailed detector design studies are currently underway.

In light of the IceCube events, the ability to trace showers back to their origins is very important. Simulation steps are similar to Sec. 2, except one now also has fluorescence capability. Light propagation and quantum efficiencies are better, but the highlight is the much higher pixelization.

We model neutrino interactions by PYTHIA. The $\tau-v_{\tau}$ angle, $\Delta \theta_{\tau}$, is less than $0.3 \operatorname{arcmin}$ for $E_{\tau}>1 \mathrm{PeV}$, which the NTA detector design is optimized for. Second, we use GEANT4 to evaluate the deflection of $\tau$ as it propagates in the Earth, using the parametrization of Ref. [21] to estimate energy loss. We compare the energy dependence of $\beta$ particle bremsstrahlung, pair production, and photonuclear interaction to Ref. [21]. The first two processes agree well, but we find smaller values for the latter at higher energies, differing by a factor of three at $10^{8} \mathrm{GeV}$. We wrote a toy Monte Carlo, where the energy dependence of the $\beta$ particle is reproduced to within $\pm 30 \%$.

Using TAUOLA and taking account of $\tau$ polarization, the deflection angle due to $\tau$ decay is less than 1 arcmin for secondary particle energy above $13 \mathrm{TeV}$. The probability for $>1$ arcmin deflection for $\mathrm{PeV} \tau \mathrm{s}$ is very small. We adopt this assumption throughout our air-shower analysis. Finally, the hadron air-shower direction is evaluated using CORSIKA. We compare the direction of the parent particle $\left(\pi^{ \pm}\right)$at shower max to that of $e^{ \pm}$, the dominant producers of Cherenkov photons. The angle between average direction of $e^{ \pm}$and the parent particle is found to be within $0.1^{\circ}$ at $1 \mathrm{PeV}$. The energy resolution is found, by MC simulation, to be several $10 \%$.

We conclude that the arrival direction of PeV-scale $v_{\tau}$ s is within $0.1^{\circ}$ of the direction of the generated hadron air-shower. 

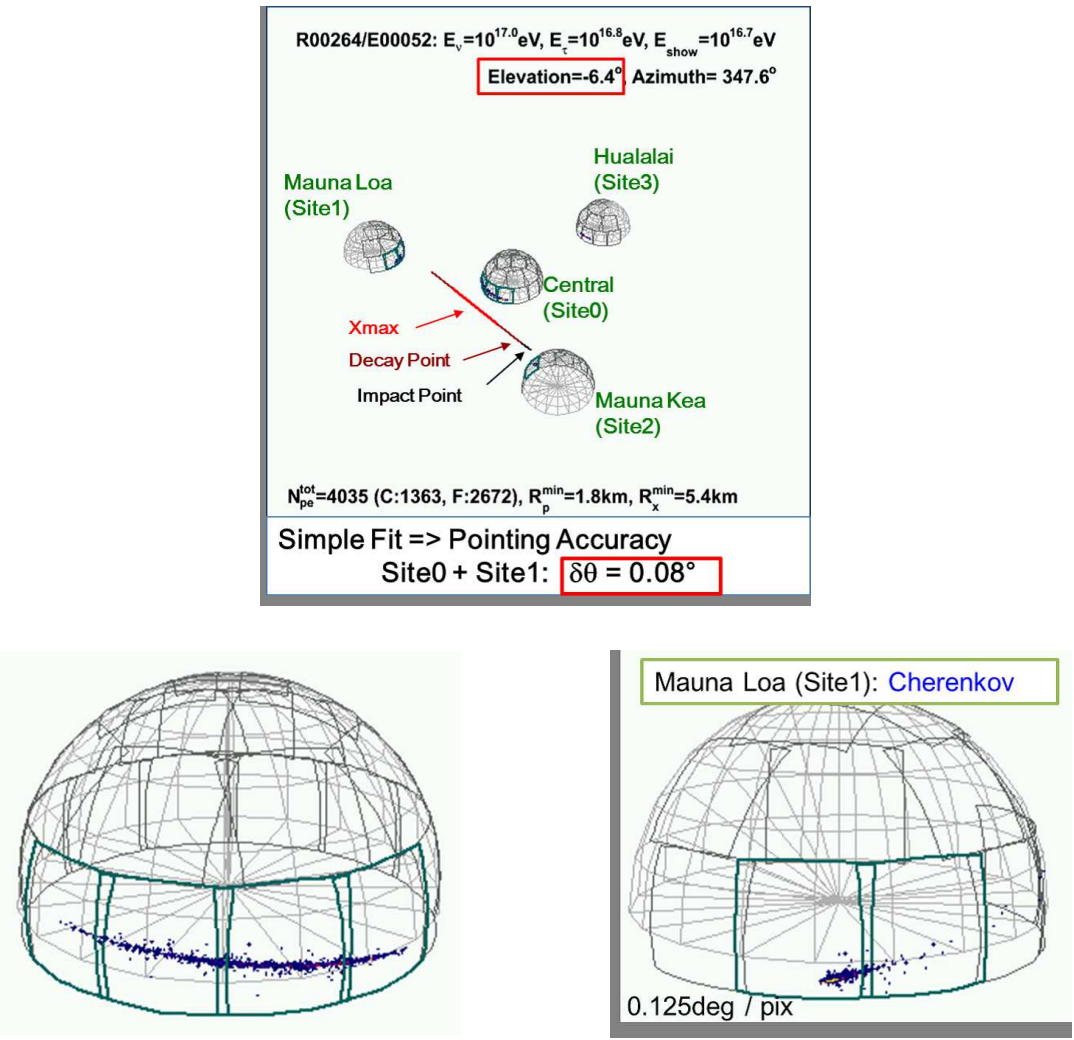

Figure 5: A simulated $\tau$ shower event generated by primary $v_{\tau}$ with $E_{v_{\tau}}=10^{17} \mathrm{eV}$ : (top) Global hit map view; (bottom left) fluorescence shower image taken by Site0, and (bottom right) Cherenkov image taken by Site1. The trigger pixel and fine image pixel FOVs are $0.5^{\circ} \times 0.5^{\circ}$ and $0.125^{\circ} \times 0.125^{\circ}$, respectively.

\section{Performance and Sensitivity}

To simulate performance of NTA detector, we assume $32^{\circ} \times 32^{\circ}$ FOV for DU, $0.5^{\circ} \times 0.5^{\circ} \mathrm{FOV}$ for trigger pixel, and $0.125^{\circ} \times 0.125^{\circ}$ FOV for image sensor pixel. From Table 1 , Site 0 consist of 12 DUs, covering the lower elevation solid angle of $\pi \mathrm{sr}$ (can be extended to full-sky coverage). The remaining sites have 6 DUs in the lower elevation angle covering $\pi / 2$ sr. The bottom edge of the lower elevation angle region is defined to be $-9^{\circ}$ (below the horizon).

In our simulation program (see LOI for slightly more detail), we take density profile of Earth and $v_{\tau}$ distribution from CTEQ4, input inelasticity parameter and parameterize energy loss in Earth. We use $\tau$ decay from TAUOLA and air-shower generation of Gaisser-Hillas, and take constant $v_{\tau}$ energy fraction of $40 \%$ (lab frame average) from $\tau$ decays, ignoring the energy distribution (error found negligible). For detector simulation, we incorporate light collection and throughput with simplified trigger logic. Event reconstruction is not yet implemented. All candidate events must satisfy the trigger conditions: number of detected photoelectrons per LC $>61 ; S / N$ estimated in track-associated $4 \times 64$ pixel box (air-shower track included) $>4$ [22]. A simulated event with primary energy $E_{v_{\tau}}=10^{17} \mathrm{eV}$, elevation angle $-6.4^{\circ}$ exiting from Mauna Kea and direction towards Mauna Loa, consistent with the above conditions, is shown in Fig. 5. A combined simple fit to Site $0 \oplus$ Site 1 gives an error for $v_{\tau}$ arrival direction at $0.08^{\circ}$. Our estimates for effective detection 

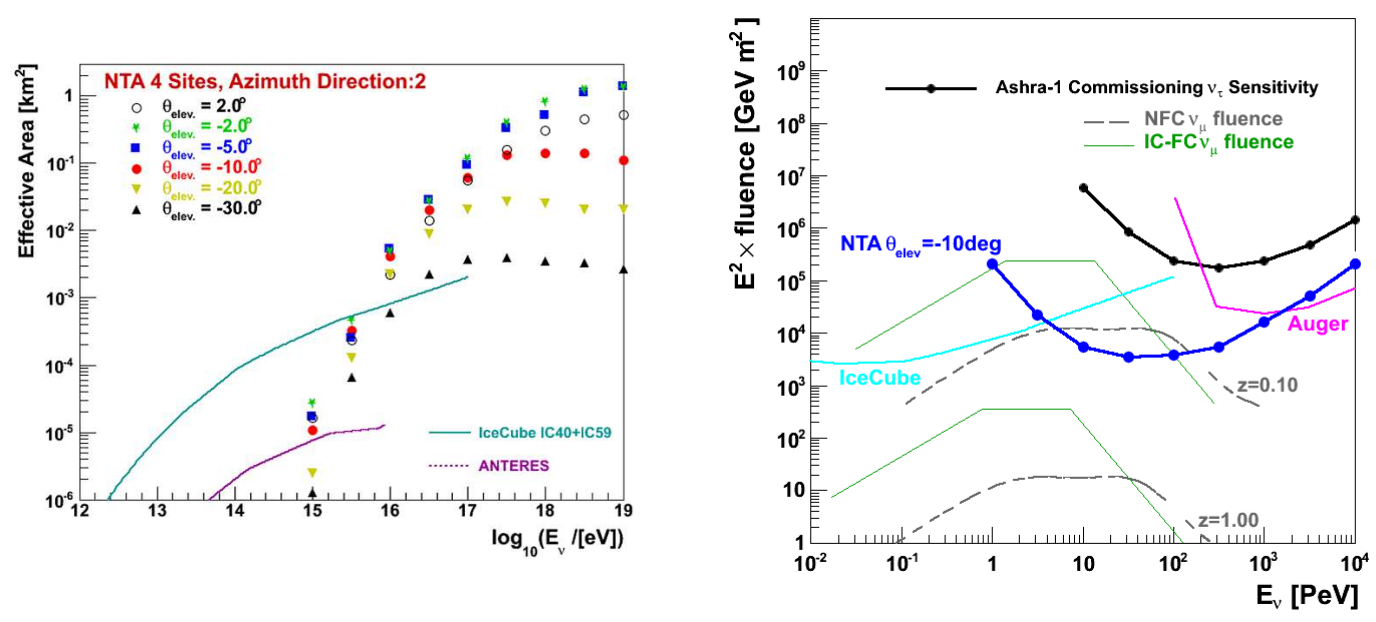

Figure 6: [left] Effective detection area w.r.t. Site0 for $v_{\tau}$ with azimuthal arrival direction towards Mauna Loa $\left(\phi_{2}\right)$, and dip angles $2.0^{\circ},-2.0^{\circ},-5.0^{\circ},-10.0^{\circ},-20.0^{\circ}$, and $-30.0^{\circ}$; [right] Feldman-Cousins $90 \%$ CL differential sensitivities for various LC designs and $\theta_{\mathrm{elev}}=-10^{\circ}$, in comparison with (earlier) published sensitivities of IceCube and Auger, together with theoretical models (dashed lines for Ref. [24]).

area for $v_{\tau}$ from point source is illustrated for Mauna Loa site for various dip angles on left side of Fig. 6 (similar result for Hualalai site). The effective detection area turns on sharply above PeV.

The IceCube PeV neutrino events clearly call for higher sensitivity searches with more accurate pointing ability. In particular, the $\mathrm{PeV}$ to $\mathrm{EeV}$ window should be measured. NTA fits the bill. Note that IceCube events do not [23] preclude the possibility of additional supra-PeV sources. We show on right side of Fig. 6 the differential sensitivity for NTA, compared side by side with IceCube and Auger capabilities (for illustration only). The solid theoretical fluence curve is ruled out by IceCube, but subsequent models for GRB neutrino flux, e.g. Ref. [24], can be probed by NTA. Given the PeV neuntrino events observed by IceCube, this search is now mandatory.

The power of NTA is to survey $v_{\tau}$ point source objects with the best-yet sensitivity in the detection solid angle for $v_{\tau}$ defined as $-30^{\circ}<\theta_{\text {elev }}<0^{\circ}$ and $0^{\circ}<\phi_{\text {azi }}<360^{\circ}$, and for $10 \mathrm{PeV}<$ $E_{v_{\tau}}<1 \mathrm{EeV}$. Fig. 6 (right) shows that the NTA survey depth can reach $z \lesssim 0.15$, of order 2 billion lightyears. The location of NTA on Hawaii Big Island allows us to survey the Galactic Center (GC) for more than several hundred hours each year, assuming the standard 10-20\% duty cycle.

NTA sensitivity for diffuse $v_{\tau}$ flux (maroon squares) for 3 year $\left(\sim 9.5 \times 10^{6} \mathrm{~s}\right)$ observation is given in Fig. 7, assuming duty cycle of $10 \%$ and trigger conditions as above. We refer further discussion on angular resolution and background simulation to the LOI [20].

As can be seen from Fig. 6 (right) and Fig. 7, although the Auger and IceCube results shown may not be the latest, their trend is indicated. Auger has studied its capabilities with UHE- $v$ s. Because of the already fixed Auger detector configuration, its best sensitivity is around $10^{18} \mathrm{eV}$ [25], complementary to that of IceCube around $10^{15} \mathrm{eV}$. But, the fact that IceCube sees several astro- $v \mathrm{~s}$ at $\mathrm{PeV}$ energy, one expects a spectrum to be probed between IceCube and Auger sensitivities. This is precisely where NTA fits in, with great pointing accuracy to pick out point sources within 2 


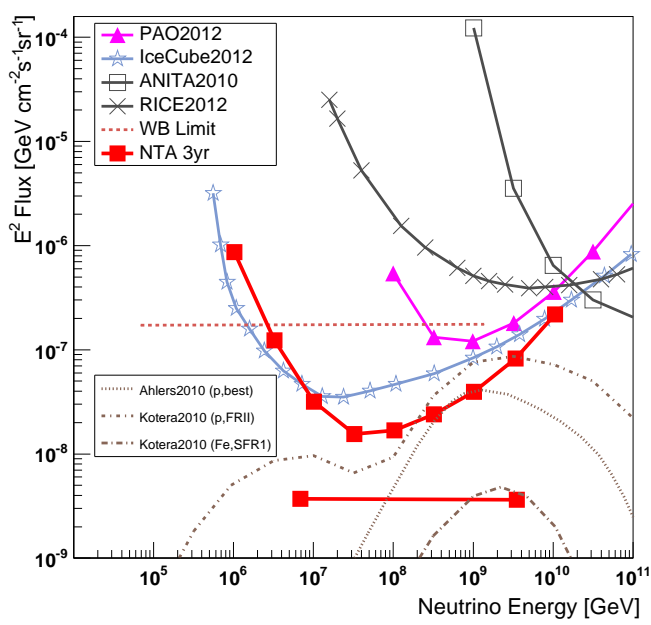

Figure 7: NTA 3 year diffuse sensitivity (red squares), together with neutrino flux models and related efforts.

Glyrs. After all, the real target is not the diffuse source, such as GZK- $v$ s (though partially probed, see Fig. 7), but "nearby" astrophysical Point Sources. Direct observation of such sources with the ES- $v_{\tau}$ technique would reveal the existence of hadron acceleration mechanisms, and open a new chapter in CR and neutrino astrophysics.

\section{Towards a New Collaboration}

We estimate that a minimum of 30 Detector Units (DUs) are needed for NTA, distributed over four mountain sites on Hawaii Island, assuming $32^{\circ} \times 32^{\circ}$ FOV for each DU. The estimated cost per DU (4 LCs plus trigger and readout), based on Ashra-1 experience, is $\sim 100 \mathrm{M}$ yen. Allowing for some infrastructure and site preparation, but not running and maintenance costs, a crude cost estimate is $5000 \mathrm{M}$ yen for the construction of NTA. NTA would eventually be a Collaboration consisting of half a dozen or more countries.

As we design the NTA instruments and explore site options, collaboration organization is still primitive. One would probably need a major funding contribution at more than $50 \%$ total cost to start attracting international collaborators, and only then (when some manpower has materialized) would one be able to seriously devise a schedule towards the scientific goal. Currently, we have a small International Executive Board (IEB) of national representatives from Japan, Taiwan and U.S., with national affairs handled domestically. Initial meetings started since late 2012, but other groups are invited to join. The time frame for the proposed project will be determined both by budgetary and scientific considerations. 2015 would be the pivotal year to not only discuss the design and plans of the project, but also major decisions on hardware implementation towards the Project Proposal. A workshop, VHEPA2016, would be held at Hawaii-Manoa in early 2016. Funding efforts would likely take 2 more years. If Japanese core funding is received in time, we hope to start experimental operations using at least part of Site 0 and Site1 by 2018. Ashra-1 would 
continue to run for both testing and scientific purposes. The expected construction time for full NTA would be of order 5 years.

In conclusion, the scientific goal of "Clear Discovery and Identification of Nonthermal Hadronic Processes in the Universe, be it Galactic, Extragalatic, or Cosmogenic." is reachable. A Collaboration, the NTA, is needed to pin down, and pinpoint, the IceCube PeV astro- $v$ events.

\section{References}

[1] M.G. Aartsen et al. [IceCube Collaboration], Phys. Rev. Lett. 111 (2013) 021103; ibid. 113 (2014) 101101.

[2] See G.W.-S. Hou, arXiv:1409.0477 [astro-ph.IM], talk presented at VHEPA2014 workshop (Tokyo) for a more personal discussion.

[3] Y. Fukuda et al. [Super-Kamiokande Collaboration], Phys. Rev. Lett. 81, 1562 (1998).

[4] For a concise and readable description of recent IceCube highlights, see the cover story article by F. Halzen and S. Klein, December 2014 issue of CERN Courier. More background material can be found in their earlier Phys. Today 61 N5 (2008) 29.

[5] See webpage http://www.auger.org/.

[6] K. Greisen, Phys. Rev. Lett. 16 (1966) 748; G.T. Zatsepin and V.A. Kuz'min, JETP Lett. 4 (1966) 78 [Pisma Zh. Eksp. Teor. Fiz. 4 (1966) 114].

[7] G.W.-S. Hou and M.A. Huang, astro-ph/0204145.

[8] D. Fargion, [astro-ph/0002453]. This paper eventually appeared as Astrophys. J. 570 (2002) 909.

[9] J.L. Feng, P. Fisher, F. Wilczek and T.M. Yu, Phys. Rev. Lett. 88 (2002) 161102.

[10] F. Vannucci, hep-ph/0106061.

[11] M. Sasaki, Y. Asaoka, and M. Jobashi, Astroparticle Physics 19 (2003) 37.

[12] M. Sasaki, J. Phys. Soc. Jpn. 77SB (Suppl. B) (2008) 83, presented at VHEPA-3 meeting held March 2003.

[13] S.W. Barwick, Phys. Scripta T 85 (2000) 106.

[14] M. Sasaki, Proc. of ICRR2000 Satellite Symposium: Workshop of Comprehensive Study of the High Energy Universe.

[15] M. Sasaki, A. Kusaka and Y. Asaoka, Nucl. Instrum. Meth. A 492 (2002) 49.

[16] Y. Asaoka, M. Sasaki, Nucl. Instrum. Meth. A 647 (2011) 34.

[17] M. Sasaki, Y. Asaoka and M. Jobashi, Nucl. Instrum. Meth. A 501 (2003) 359.

[18] Y. Aita et al., Astrophys. J. 736 (2011) L12.

[19] More results are given in talk by M. Sasaki, this proceedings.

[20] M. Sasaki and G.W.-S. Hou, arXiv:1408.6244 [astro-ph].

[21] S.I. Dutta, M.H. Reno, I. Sarcevic and D. Seckel, Phys. Rev. D 63 (2001) 094020.

[22] M. Sasaki, J. Phys. Soc. Jpn. 70 (Suppl. B) (2001) 129.

[23] M.G. Aartsen et al. [IceCube Collaboration], Phys. Rev. D 91, 022001 (2015).

[24] S. Hümmer, P. Baerwald and W. Winter, Phys. Rev. Lett. 108, 231101 (2012).

[25] P. Abreu et al. [Pierre Auger Collaboration], Astrophys. J. 755, L4 (2012). 